\title{
Does liquid-based cytology really offer any advantage?
}

\author{
Alistair R W Williams
}

A recent paper ${ }^{1}$ reported a systematic evaluation of the literature comparing liquid-based cytology (LBC) and conventional cervical cytology, and has concluded that there is no evidence that $\mathrm{LBC}$ reduced the proportion of unsatisfactory slides or detected more high-grade lesions. To many in the UK, where LBC has replaced conventional cytology or is in the process of doing so, these may be surprising conclusions. It is worth looking at the quality of evidence in the literature, the methods used in this meta-analysis, and possible reasons for the apparent discrepancy in this study's findings with the experience of some laboratories in the UK.

A total of 147 reports retrieved by computerised literature search and citation review were scrutinised and assessed for quality. The criteria for quality may not seem unreasonably stringent - an "independent randomised sample study, with verification by a masked reference standard, of at least all positive slides" - however this limited the number of studies that could be evaluated to a total of 56. These were further stratified into high, medium or low quality, on the basis of study design, setting, reference standards, masking of paired tests, and so on. Clearly the quality of evidence in the literature leaves something to be desired. For example, there is only one small (underpowered) randomised controlled trial, only five studies were judged to be of high quality, and only four provided enough data to allow estimation of sensitivity and specificity and comparison of test accuracy. It is interesting that these criteria led to exclusion of many large studies such as the UK National Institute for Health and Clinical Excellence (NICE) report, a pilot which involved 178000 slides. $^{2}$

In the depleted pool of studies that remained, almost no difference was found in the percentage of unsatisfactory slides between LBC and conventional cytology, and studies graded as high quality showed only a small increase in slides classified as high-grade abnormality, which was not apparent in lower quality studies. So, using these criteria, the available evidence appears to show little or no benefit of LBC.

It is undeniable that the quality of the evidence in the literature is variable. However, there are questions that may be asked of the authors about their own methods and conclusions. They have excluded from all analysis over $60 \%$ of relevant studies in the literature, but it is hard to believe that it is not possible to derive meaningful conclusions from these publications. Of course we all recognise that many studies are subject to all sorts of bias; but in my view, it is the task of authors setting out to perform such a meta-analysis to identify deficiencies, analyse the sources of bias, and reach conclusions. For

J Fam Plann Reprod Health Care 2006; 32(3): 149

Department of Pathology, University of Edinburgh, Royal Infirmary of Edinburgh, Edinburgh, UK

Alistair R W Williams, MD, FRCPath, Senior Lecturer/Honorary Consultant in Pathology

Correspondence to: Dr Alistair R W Williams, Department of Pathology, University of Edinburgh, Royal Infirmary of Edinburgh, 51 Little France Crescent, Edinburgh EH16 4SA, UK.

E-mail: a.williams@ed.ac.uk very good practical reasons there will never be enough randomised controlled clinical trials in areas such as cervical cytology, but we need to make best use of what evidence is available. The conclusions from this metaanalysis are based on a reduced subset of studies that have wide differences in setting that make comparisons between them highly questionable.

For example, one wonders if it is valid to compare directly results of different diagnostic classifications [e.g. the Bethesda System and the British Society for Clinical Cytology (BSCC) classification]. Was account taken of the widely varying criteria between studies used to classify a slide as unsatisfactory? In the UK, criteria for smear adequacy have long been much more stringent than in many other countries. This is a crucial point, as the decision to implement LBC within a screening programme may well hinge on the inadequate rate existing in that programme. It is clear that LBC will not improve adequacy much in a setting where the inadequate rate is around 2\% already, such as in The Netherlands. However, in England in 2004-2005 (almost all conventional slides), $75 \%$ of all laboratories had inadequate rates of between $6 \%$ and $12 \%$, and almost $10 \%$ had over $12 \%$.

In my own laboratory, our experience with LBC has been very positive. Since the introduction of LBC in 2003 , the inadequate rate has fallen from over $12 \%$ with conventional cytology to less than $2 \%$, with substantial benefit to the women screened, the smear takers, colposcopy clinics and the cytopathology laboratory itself. ${ }^{3}$ Productivity in primary screening has improved so much that backlogs - previously unacceptably high with up to 10-week turnaround times at their worst - have disappeared despite screening staff numbers continuing to fall. The pick-up rate of high-grade abnormality is at least as good as it was with conventional cytology, and the positive predictive value for high-grade abnormality has improved.

Such dramatic improvements will probably not be seen where inadequacy rates are lower. It is very important that we do not conclude that LBC and conventional cytology are equivalent, and that LBC offers no advantage - this will vary according to the setting. New technology always needs to be evaluated properly, and at present the published studies on LBC are variable in quality. However, if authors of meta-analyses restrict themselves to selected subsets of studies ignoring substantial parts of the literature (flawed as it may be), it is not surprising that sometimes they will come to conclusions that are at odds with reality.

Statements on funding and competing interests

Funding. None identified.

Competing interests. None identified.

References

1 Davey E, Barratt A, Irwig L, Chan SF, Macaskill P, Mannes P, et al. Effect of study design and quality on unsatisfactory rates, cytology classifications, and accuracy in liquid-based versus conventional cervical cytology: a systematic review. Lancet 2006; 367: 122-132.

2 Karnon J, Peters J, Chilcott J, McGoogan E. Liquid-based Cytology in Cervical Screening: An Updated Rapid and Systematic Review. 2003. http://www.nice.org.uk/pdf/LBC_Assessmentreport.pdf [Accessed 5 May 2006]

3 Williams AR. Liquid-based cytology and conventional smears compared over two 12-month periods. Cytopathology 2006; 17: $82-85$. 